\title{
Uncertainty in modelled soil organic carbon changes under various cropping systems in Australian cropland
}

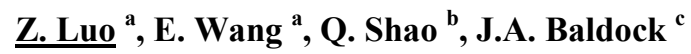 \\ ${ }^{a}$ CSIRO Land and Water, Canberra, ACT 2601, Australia; ${ }^{b}$ CSIRO Computational Informatics, Floreat, WA \\ 6014, Australia; ${ }^{c}$ CSIRO Land and Water, Glen Osmond, SA 5604, Australia \\ Email: zhongkui.luo@,csiro.au
}

\begin{abstract}
Agricultural soils have been suggested to sequester soil organic carbon (SOC) through adopting conservation agricultural practices such as residue retention, no-till and increasing cropping complexity. At regional or continental scales, however, it is a significant challenge to quantify the changes in SOC stock and the corresponding uncertainty associated with variation of cropping systems and environmental conditions at high spatiotemporal resolution. Here, our aim was to predict changes in SOC stock after adopting different cropping systems under optimal management for sequestering soil carbon (i.e., no nutrient deficiency and $100 \%$ residue retention) and quantify the relevant uncertainties at regional scale. Using the farming systems model APSIM, we simulated changes in SOC stocks for a 20-year period from 1990 to 2010 under a total of 59 cropping systems at 613 references sites across the Australian cereal-growing regions. These cropping systems were identified based on GRDC agro-ecological zones through expert consultation. To further understand the effects of cropping system in terms of carbon input on SOC dynamics, those cropping systems were divided into three categories to represent low-, medium- and high-input cropping systems in terms of carbon input. The simulation results indicated that, on average, the Australian agricultural soils could gain $0.19 \mathrm{t} \mathrm{C} \mathrm{ha}^{-1} \mathrm{yr}^{-1}$ under optimal management. However, the predicted change in SOC stocks had high variability among the three carbon-input categories. Generally, cropping systems with higher carbon input had higher efficiency for reducing SOC losses or enhancing SOC gains compared to lower carbon-input systems. For the same category of cropping system, its ability to reduce SOC losses or enhance SOC gains varied across difference GRDC zones. For example, in Qld Central zone where has higher temperature, the SOC experienced loss regardless of cropping system. In SA Vic High Rainfall zone where has lower temperature and higher rainfall, the SOC showed increase. This result indicated the importance of local soil and climate conditions to regulate the SOC dynamics under different cropping systems.
\end{abstract}

A Monte Carlo approach was applied to assess the uncertainty induced by cropping system and scaling of point results to GRDC zone. Averaged across all representative cropping systems, the predicted mean SOC change was $-0.1 \mathrm{t} \mathrm{C} \mathrm{ha}^{-1} \mathrm{yr}^{-1}$ with the $95 \%$ confidence interval ranging from -0.22 to $+0.007 \mathrm{t} \mathrm{C} \mathrm{ha}^{-1} \mathrm{yr}^{-1}$ in Qld Central. In NSW NW/Qld SW, the predicted SOC change was zero with the $95 \%$ confidence interval ranging from -0.05 to $+0.05 \mathrm{t} \mathrm{C} \mathrm{ha}^{-1} \mathrm{yr}^{-1}$. In other zones, the predicted SOC change was positive. In Vic High Rainfall zones, the SOC change reached the greatest increase of $+0.44 \mathrm{t} \mathrm{C} \mathrm{ha}^{-1} \mathrm{yr}^{-1}$ with the $95 \%$ confidence interval ranging from +0.22 to $+0.66 \mathrm{t} \mathrm{C} \mathrm{ha}^{-1} \mathrm{yr}^{-1}$. In Western Australia, the predicted SOC change was generally positive across all representative cropping systems. There was significant difference between different cropping system categories. In general, cropping systems with higher carbon input could reduce the SOC losses or enhance SOC gains compared to cropping systems with lower carbon input. In three zones of Western Australia (WA Northern, WA Eastern and WA Central), however, the predicted average SOC change under high-input cropping systems was lower than that under medium-input cropping systems. We further calculated the contribution of cropping systems and scaling to overall uncertainty. The simulation indicated that the variability of cropping system accounted for $\sim 30 \%$ of the overall uncertainty. The greatest contribution of cropping system change to uncertainty in simulated SOC $(>60 \%)$ was observed in three GRDC zones, i.e., NSW NW/Qld SW, NSW NW/Qld SE, and WA Northern. Our results suggested that the uncertainty in scaling of point results to regional scale is dominant in the overall uncertainty. More detailed soil databases and information on cropping systems are needed for reliable prediction of SOC dynamics in agricultural soils at regional scale.

Keywords: APSIM, carbon input, crop sequence, Monte Carlo simulation, soil carbon change, uncertainty analysis 


\section{INTRODUCTION}

Cultivation of natural soils has induced $20-70 \%$ declines in soil organic carbon (SOC) in Australia (Luo et al., 2010, Dalal \& Chan, 2001). Conservation agricultural practices have been widely suggested to increase SOC in agricultural soils thereby mitigating greenhouse gas emission (Dalal and Chan, 2001, Luo et al., 2010). In addition, many field experimental studies showed that SOC could be significantly affected by the type of cropping systems with the varying impact depending on rotation types and environmental conditions. Accurate quantification of the effect of cropping system on SOC is however a challenge due to the diversity of cropping systems. In Australia, Unkovich et al. (2009) suggested that a total of 22 crops should be included in carbon accounting system in order to account for $>99 \%$ of the sowing area in all states. But how these crops will be planted in crop rotations vary in time and space. Therefore, more reliable prediction of SOC stock will require quantification of the uncertainty associated with the input of carbon to soil derived from variations in cropping systems and environmental conditions at the desired spatial and temporal resolutions.

In this study, we used the widely tested process-based APSIM model (Keating et al., 2003) to conduct simulations on change in SOC stocks under 59 cropping systems at 613 reference sites located in Australia's cereal-growing regions. Those cropping systems included nine major crops sown in Australia, which were identified based on agro-ecological zones defined by Grain Research and Development Corporation (GRDC). Our objective was to estimate potential changes in SOC stock in each agro-ecological zone under the most representative cropping systems together with optimal management, i.e., $100 \%$ residue retention and no nutrient deficiency. In addition, we assessed the uncertainty of estimated changes in SOC stock induced by possible changes in cropping system and scaled the point results to GRDC zones.

\section{MATERIALS AND METHODS}

\subsection{Data sources}

The soil profile data for 613 reference sites were obtained from the Agricultural Production Systems Research Unit) database and the sites are distributed throughout the study region (available at http://www.asris.csiro.au/themes/model.html, Fig 1). These data contain the full soil profile characterization required to run the APSIM model. Twenty years of daily weather data from 1990 to 2010 was acquired for the meteorological station located closest to each of the 613 soil sites from the SILO Patched Point Dataset (http://www.longpaddock.qld.gov.au/silo/).

\subsection{Cropping systems}

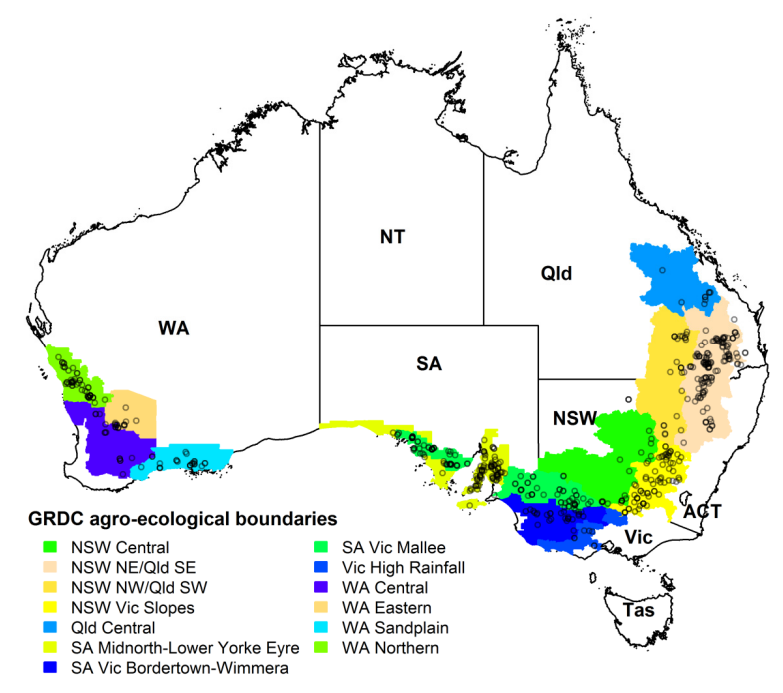

Figure 1 The location of the 613 reference soil sites (black open circles) and the boundary of the 13 GRDC agro-ecological zones in Australia. ACT, Australian Capital Territory; Qld, Queensland; NSW, New South Wales; NT, Northern Territory, SA, South Australia; Tas, Tasmania; Vic, Victoria; WA, Western Australia. 
Luo et al., Uncertainty in modelled soil organic carbon changes under various cropping systems in Australian cropland

Table 1 The number of soil sites $\left(n_{s}\right)$ and cropping systems $\left(n_{c}\right)$ in each zone, and the percentage of total uncertainty associated with the cropping system and scaling of results from points to the GRDC zone.

\begin{tabular}{lcccc}
\hline GRDC zones & $n_{s}$ & $n_{c}$ & Cropping system (\%) & Scaling (\%) \\
\hline Qld Central & 9 & 6 & 27 & 73 \\
NSW NW/Qld SW & 27 & 10 & 60 & 40 \\
NSW NE/Qld SE & 137 & 7 & 62 & 38 \\
NSW Central & 22 & 13 & 45 & 55 \\
NSW Vic Slopes & 68 & 7 & 33 & 67 \\
SA Midnorth-Lower Yorke Eyre & 110 & 4 & 16 & 84 \\
SA Vic Mallee & 30 & 4 & 3 & 97 \\
SA Vic Bordertown-Wimmera & 90 & 3 & 52 & 48 \\
Vic High Rainfall & 5 & 2 & 24 & 76 \\
WA Sandplain & 24 & 3 & 20 & 80 \\
WA Northern & 59 & 4 & 61 & 39 \\
WA Eastern & 7 & 4 & 25 & 75 \\
WA Central & 25 & 2 & 12 & 88 \\
\hline
\end{tabular}

Information on cropping systems was collated based on the GRDC agro-ecological zones. These GRDC zones were classified according to the dominant crop rotations and agricultural management regimes as well as edaphic and climatic conditions. Five of the 18 zones were excluded in this study due to either limited cropping areas or a lack of soil profile data. The remaining 13 zones cover most of the whole eastern and western grain growing belt, and more than $95 \%$ of the cropped area in Australia (Fig. 1). The number of soil sites in each zone ranged from 5 to 137 (Table 1).

No detailed records on the crop rotations were available due to the spatial variations resulting from significant inter-annual climate variability and frequent resource limitation at farm level. Little evidence existed to suggest that any farmers would continuously implement a fixed crop rotations over time. Therefore, for the purpose of modelling SOC dynamics across the grain regions, representative crop rotations (or crop-pasture rotations) were constructed based on consultation with agronomists and agricultural consultants and on collation of the most frequently reported cropping rotations in each of the GRDC zones. Each of the rotations constructed represented a fixed rotation of crops and/or pastures. Overall, nine crops, i.e., wheat, barley, canola, lupin, chickpea, fieldpea, fababean, sorghum and cotton, were included in those rotations. They were the major crops and account for the majority of the total cropping area of broadacre crops in Australia (Unkovich et al., 2009). Lucerne was assumed in the crop-pasture rotations to represent the perennial pasture.

Those representative cropping systems were further divided to three categories, i.e., low-input, medium-input and high-input cropping systems in terms of carbon input (IPCC, 2003; Wang et al., 2013). Low-input cropping systems were characterised by low residue return due to removal of residues, frequent barefallowing or production of crops yielding low residues (e.g., cotton). Medium-input rotations included annual cropping with cereal where all crop residues were returned to the field. High-input rotations included significantly greater crop residue yields, use of green manure, cover crops, improved vegetated fallows, frequent use of perennial grasses in annual crop rotations (IPCC, 2003). The number of cropping systems in each zone is shown in Table 1. More details on the cropping systems in each zone can be found in Wang et al. (2013).

\subsection{APSIM simulations}

APSIM v7.3 was used to predict the changes in SOC stocks under the identified cropping systems and under a simplified continuous wheat system representing an annual cereal cropping system. APSIM simulates crop growth and soil processes on a daily time-step in response to climate (i.e., temperature, rainfall, and radiation), soil water availability, and soil nutrient status (Keating et al., 2003, Probert et al., 2005, Luo et al, 2011). APSIM allows flexible specification of management options like crop and rotation type, tillage, residue management, fertilization and irrigation. The ability of APSIM to simulate SOC dynamics has been verified under different cropping systems and management in a number of studies (Probert et al., 2005, Huth et al., 2010, Luo et al., 2011). 
For each of the crop rotations simulated, crops were sown every year depending on rainfall and soil water content, which varied between regions and with the crops included in the rotation. Cultivars were assigned according to sowing date - the earlier the sowing date, the later the maturity type of the crop cultivar. For simplicity, three cultivars for each crop representing early, middle and later maturity cultivars were selected from the listed default cultivars in the parameter files released with the APSIM v7.3 model for each zone. For perennial lucerne, however, only one cultivar (i.e., trifecta) was used. Crop residues (stem plus leaf) after harvest were retained in the system. Lucerne was sown and removed after harvest and before sowing of annual crops in the corresponding rotations, respectively. Harvest to the height of $10 \mathrm{~cm}$ was assumed to occur each time lucerne reached the flowering stage.

\subsection{Data assessment and uncertainty analysis}

Soil organic carbon stocks in the top $0.3 \mathrm{~m}$ soil profile were simulated every year for each soil site and each cropping system. The change in SOC stocks $\left(C_{c}\right)$ was calculated as the average annual change in SOC stock during the 20-year simulation for each zone, and for each cropping system category (i.e., low-, medium-, and high-input) and putting all cropping systems together (i.e., all input). The uncertainty analysis was conducted for individual cropping system categories. For simplicity, the cropping system category is not shown in the notation. As the number of simulations varied significantly in each GRDC zone for each cropping system, we used a Monte Carlo approach to generate an equal number of replicates for uncertainty analysis. To do this, in each GRDC zone, the mean $(\mu)$ and standard deviation $(\sigma)$ of $C_{c}$ for each cropping system category and for all cropping systems were calculated to construct a normal probability density function $N\left(\mu, \sigma^{2}\right)$ assuming the resultant values of $C_{c}$ followed a normal distribution. A Monte Carlo approach was used to generate 1000 replicates which were used to conduct an uncertainty analysis as follows. In each GRDC zone, first, an estimate $\theta$ for each Monte Carlo replicate $i$ was computed as:

$$
\theta_{i}=\frac{1}{n} \times \sum_{k} \Delta C_{k}^{i}
$$

where $n$ was the number of soil sites in the GRDC zone of interest, and $\Delta C_{k}^{i}$ the change in SOC for the $k^{\text {th }}$ soil site and sampled from the corresponding $N\left(\mu, \sigma^{2}\right)$. Then, the estimation was completed by computing the average of the Monte Carlo replicates:

$$
\theta=\frac{1}{m} \times \sum_{i=1}^{m} \theta_{i}
$$

The variance $\left(V_{l}\right)$ of the $m$ (i.e., 1000) Monte Carlo replicates includes uncertainties associated with the model inputs and structure (Ogle et al., 2010), i.e., cropping systems in this study, and was calculated as:

$$
V_{1}=\frac{\sum_{i=1}^{m}\left(\theta_{i}-\theta\right)^{2}}{m-1}
$$

Additional uncertainty was associated with scaling of results from soil sites to the GRDC zone. A standard variance estimator for a stratified two-stage sample design $\left(V_{2}\right)$ was calculated to address this uncertainty (Ogle et al., 2010):

$$
V_{2}=\frac{\sum_{k \in S_{h}}\left(\Delta C_{k}^{*}-\frac{1}{n} \times \sum_{k \in S_{h}} \Delta C_{k}^{*}\right)^{2}}{n \times(n-1)}
$$

where $S_{h}$ was the set of soil sites in GRCD zone $h$, and $\Delta C_{k}^{*}$ was given by:

$$
\Delta C_{k}^{*}=\frac{1}{m} \times \sum_{i=1}^{m} \Delta C_{k}^{i}
$$

and was the average SOC stock change rate (across $m$ Monte Carlo replicates) for the $k^{\text {th }}$ soil site at the corresponding GRDC zone. The total variance for the change in SOC was estimated by combining the two components, i.e., $V_{T}=V_{1}+V_{2}$. The square root of the $V_{T}$ (i.e., standard deviation) was used to construct a $95 \%$ confidence interval under normality assumption. For all inputs, the contribution of the variation of cropping systems to total uncertainty was further calculated through dividing $V_{I}$ by $V_{T}$. 


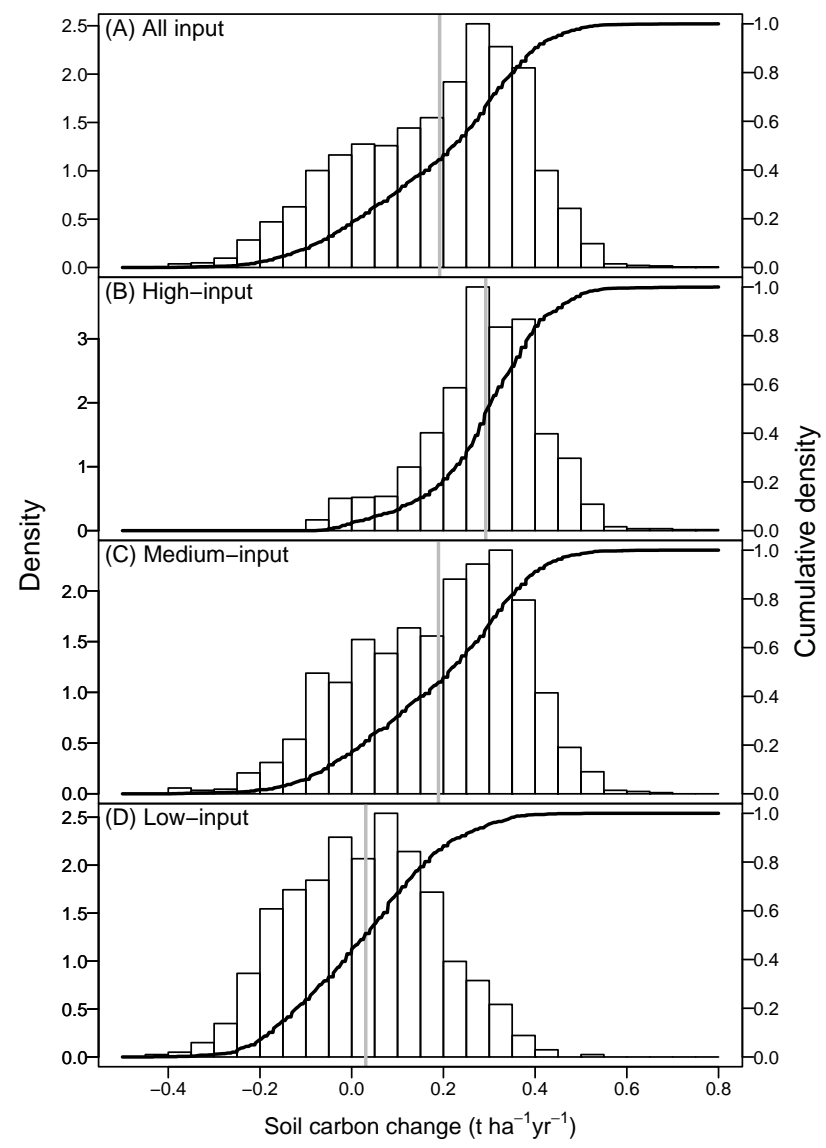

Figure 2 The probability density distribution of soil organic carbon changes under all representative cropping systems (A), high-input (B), medium-input (C) and low-input (D) rotations at the 613 reference sites. The vertical grey lines show the mean of the distribution.

\section{RESULTS}

At the national scale, averaging across all the representative cropping systems (e.g., all input in Fig. 2A ), the average predicted $C_{c}$ was $+0.19 \mathrm{t} \mathrm{C} \mathrm{ha}^{-1} \mathrm{yr}^{-1}$ with $2.5 \%, 50 \%$ and $97.5 \%$ quantiles of $-0.19,+0.23$ and +0.49 t C ha ${ }^{-1} \mathrm{yr}^{-1}$, respectively. Cropping systems under different carbon input categories had a significant effect $\left(F_{(2,3727)}=771.6, P<0.001\right)$ on the predicted $C_{c}$ (Fig. 2). Under the high-input cropping systems, the average $C_{c}$ was $+0.29 \mathrm{t} \mathrm{C} \mathrm{ha}^{-1} \mathrm{yr}^{-1}$ with $2.5 \%, 50 \%$ and $97.5 \%$ quantiles of $-0.01,+0.30$ and $+0.51 \mathrm{t} \mathrm{C} \mathrm{ha}^{-1} \mathrm{yr}^{-1}$, respectively (Fig. 2B). Under the medium-input cropping systems, this was $+0.19 \mathrm{t} \mathrm{C} \mathrm{ha}^{-1} \mathrm{yr}^{-1}$ with $2.5 \%$, $50 \%$ and $97.5 \%$ quantiles of $-0.16,+0.22$ and $+0.48 \mathrm{t} \mathrm{C} \mathrm{ha}^{-1} \mathrm{yr}^{-1}$, respectively (Fig. 2C). Under low-input systems, this was $+0.03 \mathrm{t} \mathrm{C} \mathrm{ha}^{-1} \mathrm{yr}^{-1}$ with $2.5 \%, 50 \%$ and $97.5 \%$ quantiles of $-0.25,+0.03$ and $+0.34 \mathrm{t} \mathrm{C} \mathrm{ha}^{-1}$ $\mathrm{yr}^{-1}$, respectively (Fig. 2D).

For each zone, the predicted average $C_{c}$ and its uncertainty are shown in Fig 3. Averaged across all representative cropping systems (i.e., all input), the predicted mean $C_{c}$ was $-0.1 \mathrm{t} \mathrm{C} \mathrm{ha-1} \mathrm{yr}^{-1}$ with the $95 \%$ confidence interval ranging from -0.22 to $+0.007 \mathrm{t} \mathrm{C} \mathrm{ha}^{-1} \mathrm{yr}^{-1}$ in Qld Central (Fig. 3A). In NSW NW/Qld $\mathrm{SW}$, the predicted $C_{c}$ was zero with the $95 \%$ confidence interval ranging from -0.05 to $+0.05 \mathrm{t} \mathrm{C} \mathrm{ha}^{-1} \mathrm{yr}^{-1}$ (Fig. 3B). In other zones, the predicted $C_{c}$ was positive. Generally, in southern and eastern Australia, the simulated $C_{c}$ increased roughly following the rainfall amount from northwest to southeast. In Vic High Rainfall zones, the $C_{c}$ reached the greatest increase of $+0.44 \mathrm{t} \mathrm{C} \mathrm{ha}^{-1} \mathrm{yr}^{-1}$ with the $95 \%$ confidence interval ranging from +0.22 to $+0.66 \mathrm{t} \mathrm{C} \mathrm{ha}^{-1} \mathrm{yr}^{-1}$ (Fig. 3I). In Western Australia, the predicted $C_{c}$ was generally positive across all representative cropping systems (Fig.3J, K, L and M). There was significant difference between different cropping system categories (Fig. 3). In general, cropping systems with higher carbon input could reduce the SOC losses or enhance SOC gains compared to cropping systems with lower carbon input. In three zones of Western Australia (WA Northern, WA Eastern and WA Central), however, the predicted average $C_{c}$ under high-input cropping systems was lower than that under medium-input cropping systems (Fig. 3K, L and M). 
The contribution of cropping system to uncertainty ranged from $3 \%$ to $62 \%$ in the studied 13 agro-ecological zones (Table 1). In NSW NW/Qld SW, NSW NW/Qld SE, and WA Northern, the cropping system accounted for more than $60 \%$ of the overall uncertainty. In SA Vic Mallee and WA Central, the cropping system accounted for less than $20 \%$ of the over uncertainty. In other zones, the cropping systems accounted for 20 $60 \%$ of the overall uncertainty. Averaging across all zones, the contribution of cropping system to overall uncertainty was $34 \%$.

\section{DISCUSSION AND CONCLUSIONS}

Our simulation results suggested that soils in Australia's croplands may be able to accumulate SOC if best management practices could be adopted, i.e., optimal application of fertilizers and $100 \%$ residue retention,

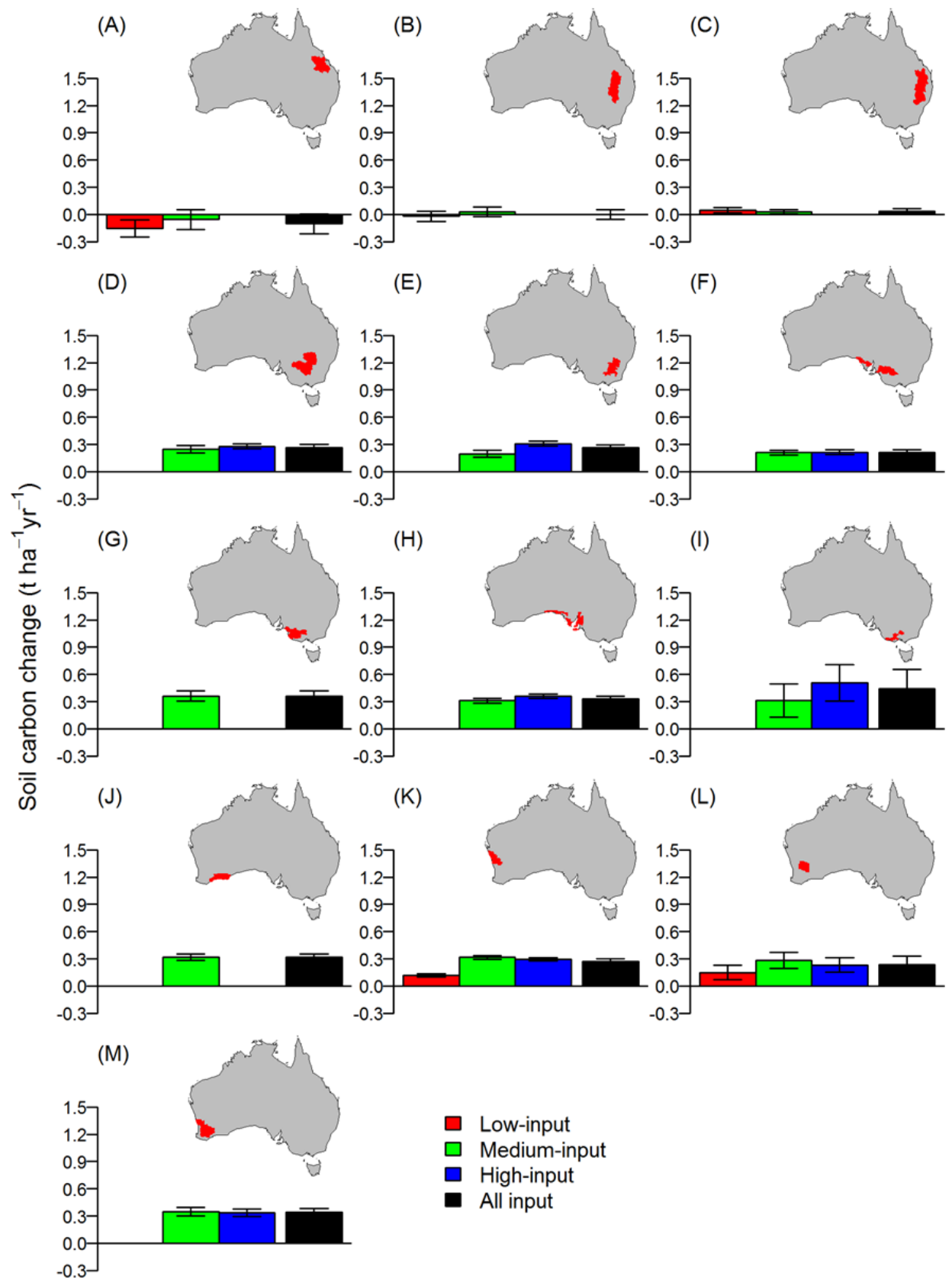

Figure 3 Mean annual soil organic carbon change under different cropping systems in terms of carbon inputs with optimal management in the 13 agro-ecological zones of Australia. (A), Qld Central; (B) NSW NW/Qld

SW; (C) NSW NE/Qld SE; (D), NSW Central; (E), NSW Vic Slopes; (F), SA Vic Mallee; (G), SA Vic Bordertown-Wimmera; (H), SA Midnorth-Lower Yorke Eyre; (I), Vic High Rainfall; (J), WA Sandplain; (K), WA Northern; (L), WA Eastern; (M), WA Central. Bars show the 95\% confidence interval. 
under representative cropping systems. At the national scale, the average predicted change in SOC stocks was $+0.19 \mathrm{t} \mathrm{C} \mathrm{ha}^{-1} \mathrm{yr}^{-1}$ during the simulated period. This result suggests that agricultural soils of Australia may provide a net sink of atmospheric carbon dioxide if conservation agricultural practices are adopted consistent with statements made by Dalal \& Chan (2001) and Luo et al. (2013). However, there was large uncertainty in the predicted SOC change due to variations in cropping systems and when scaling the point results to regional scale. For example, the predicted SOC change ranged from gains to large losses in those zones with higher temperature and/or lower rainfall (e.g., Qld Central). In those regions, soil water availability would limit the overall production of different cropping systems thereby carbon input to the soil.

Quantifying uncertainties is necessary to determine the confidence that can be placed in model predictions. Results indicated that at a regional scale, on average, uncertainty associated with scaling of point results was greater than the uncertainty induced by variation of cropping systems. Increasing the number of sites with detailed soil information will likely reduce uncertainty in scaling. In this study, the uncertainties associated with model structure including algorithms and parameterization (e.g., initial composition of SOC pools) were not considered. Such uncertainties have been suggested to dominate the total uncertainties associated with upscaling point results to a regional scale (Ogle et al., 2010). Long-term temporal monitoring of soil carbon stocks is required to further develop the relationship between measurement and model results help quantify uncertainty derived from model algorithms and structure.

\section{ACKNOWLEDGEMENTS}

This study is supported by funding from the Australian Government Department of Agriculture, Fisheries and Forestry (DAFF) and the Grain Research and Development Corporation (GRDC).

\section{REFERENCES}

Dalal RC, Chan KY (2001) Soil organic matter in rainfed cropping systems of the Australian cereal belt. Australian Journal of Soil Research, 39, 435-464.

Gaiser T, Abdel-Razek M, Bakara H (2009) Modeling carbon sequestration under zero-tillage at the regional scale. II. The influence of crop rotation and soil type. Ecological Modelling, 220, 3372-3379.

Huth NI, Thorburn PJ, Radford BJ, Thornton CM (2010) Impacts of fertilisers and legumes on N2O and CO2 emissions from soils in subtropical agricultural systems: A simulation study. Agriculture Ecosystems \& Environment, 136, 351-357.

IPCC (2003) Good practice guidance for land use, land-use change and Forestry. IPCC National Greenhouse Gas Inventories Programme, http://www.ipcc-nggip.iges.or.jp/public/gpglulucf/gpglulucf_contents.html.

Keating BA, Carberry PS, Hammer GL et al. (2003) An overview of APSIM, a model designed for farming systems simulation. European Journal of Agronomy, 18, 267-288.

Luo Z, Wang E, Bryan BA, King D, Zhao G, Pan X, Bende-Michl U (2013) Meta-modeling soil organic carbon sequestration potential and its application at regional scale. Ecological Applications, 23, 408-420.

Luo Z, Wang E, Sun OJ (2010) Soil carbon change and its responses to agricultural practices in Australian agro-ecosystems: A review and synthesis. Geoderma, 155, 211-223.

Luo Z, Wang E, Sun OJ, Smith CJ, Probert ME (2011) Modeling long-term soil carbon dynamics and sequestration potential in semi-arid agro-ecosystems. Agricultural and Forest Meteorology, 151, 15291544.

Ogle S, Breidt F, Easter M, Williams S, Killian K, Paustian K (2010) Scale and uncertainty in modeled soil organic carbon stock changes for US croplands using a process-based model. Global Change Biology, 16, 810-822.

Probert ME, Delve RJ, Kimani SK, Dimes JP (2005) Modelling nitrogen mineralization from manures: representing quality aspects by varying C : N ratio of sub-pools. Soil Biology \& Biochemistry, 37, 279287.

Unkovich M, Baldock J, Marvanek S (2009) Which crops should be included in a carbon accounting system for Australian agriculture? Crop \& Pasture Science, 60, 617-626.

Wang E, Luo Z, Xing H, Baldock J, Thorburn P (2013) Prediction of soil organic carbon sequestration potential at reference sites across Australian grain regions - A technical report to the Department of Agriculture, Forestry and Fisheries and the Grain Research and Development Corporation. CSIRO, Australia.

West TO, Post WM (2002) Soil organic carbon sequestration rates by tillage and crop rotation: A global data analysis. Soil Science Society of America Journal, 66, 1930-1946. 